ACTA SCIENTIFIC MEDICAL SCIENCES (ISSN: 2582-0931)

Volume 3 Issue 8 August 2019

\title{
Analysis of Operating Parameters of Waterjet for Skin Incisions
}

\author{
Abdou George* \\ Mechanical and Industrial Engineering Department, New Jersey Institute of Technology, Newark, New Jersey, USA \\ *Corresponding Author: Abdou George, Mechanical and Industrial Engineering Department, New Jersey Institute of Technology, \\ Newark, New Jersey, USA.
}

Received: July 02, 2019; Published: July 18, 2019

DOI: 10.31080/ASMS.2019.03.0360

\section{Abstract}

The paper describes the practical effects of the operating parameters in the skin incisions. In a previous study, experiments have been conducted to measure the modulus of elasticity of animal skins. Three analytical mathematical model has been developed: Waterjet Pressure, Depth of incision and Water jet Pressure/velocity. A process optimization that is based on the maximum depth of incision has been applied to relate the Waterjet Pressure model, depth of incision and process criteria, such as cutting/drilling/ debridement. Using a specific energy for the skin to be $1.43 \times 10^{5} \mathrm{MPa}$, the models have been tested using the experimental work done by Honl., et al. in 2004 . The percent error between the two values ranges between $2 \%$ and $11 \%$.

Keywords: Pressure; Velocity; Modulus of Elasticity

\section{Problem definition}

There are several tools that are used to make minimally invasive skin incisions: Traditional tools and non-traditional tools. Traditional tools include scalpels, surgical scissors and surgical air drills while non-traditional tools include $\mathrm{CO}_{2}$ laser, diathermy and harmonic scalpels. WJ technology is considered a non-traditional tool for making surgical incisions. While it is not usually used for skin incisions, it has the potentials and advantages to accomplish such task.

The depth and length of skin incisions depend on the tools that are utilized to make such incisions. The most traditional and commonly used tool for skin incision, whether for cutting or debridement, is the scalpel. A scalpel's number refers to the size of the scalpel's blade size. Each blade varies in length and shape of the cutting edge to suit different purposes. There are two main methods for skin incisions using a scalpel: press cutting and slide cutting.

Another traditional type of surgical tool used for skin cutting and debridement is the surgical scissors. Scissor blades can either be curved or straight. In order to achieve the best wound healing when using surgical scissors, the surgeon should cut exactly at the point where the blades meet. A disadvantage of the surgical scissors is they are designed primarily for right-handed users.

The surgical air drill is used for drilling holes through the skin and bone in various procedures. An advantage of the specialized air drill, which is used to drill the skull, is that it has a feedback system that is designed to stop drilling automatically once the skull is penetrated. This design prevents injuries to the brain.

$\mathrm{CO}_{2}$ laser is a non-traditional tool that is used for skin incision. The way a $\mathrm{CO}_{2}$ laser works is by vaporizing water that is found in the skin and other soft tissue. One thin layer of skin at a time is removed when using $\mathrm{CO}_{2}$ laser which causes no damages to the surrounding areas. $\mathrm{CO}_{2}$ laser enables high precision in removing the tissue while simultaneously providing sufficient hemostasis.

Diathermy, which is also known as electrocautery, is an alternative non-traditional way that is used for skin incision. Continuous high frequency current of $100 \mathrm{kHz}$ or above of sufficient voltage that ranges from 200 to $500 \mathrm{~V}$ is used for skin incision. The diathermy tool allows for faster incisions and reduced bleeding while causing less postoperative pain over a traditional scalpel. 
A newer non-traditional tool used for skin incision is the harmonic scalpel. The harmonic scalpel uses ultrasonic energy to simultaneously cut and cauterize tissues including sealing the veins. The main advantages of the harmonic scalpel is precise dissection, reliable hemostasis in addition to less lateral thermal spread.

While the non-traditional processes for skin incision tools have shown several advantages to justify their use over the traditional scalpel, surgical scissors and drills, some disadvantages still pose a hindrance. Such disadvantages include producing burning of variable depth in the tissues which may affect outcome of surgical wound. Additionally, the patients can smell their skin burning which can be unpleasant and unsettling. Furthermore, the use of intraoperative energized dissection can result in surgical smoke containing potentially carcinogenic and irritant chemicals.

A WJ tool has the same advantages of the non-traditional tools to justify using it over traditional tools for minimally invasive skin incision but without the thermal damage to the separated tissue due to its coolant ability. The technique of the WJ tool is simply moving the tool in a line to apply the pressure and the cut, rendering the main advantage of WJ incision its precision. The WJ tool has an essential advantage over laser scalpels due to its ability to control and change the water pressure during an incision making it more selective. For example, a WJ tool is able to cut liver tissue without cutting a vein. Furthermore, the WJ washes away blood which eliminates any extra tools necessary for this action which would be required in a regular cut. According to Dr. Matthew Hanna, Neuropathologist at Memorial Sloan Kettering Cancer Center, while providing the same or better precision as a traditional scalpel, WJ can augment or replace current surgical tools for skin incisions [1-15].

\section{Literature Review}

Many studies have been done using WJ technology to drill or cut bone or bone cement. A 2004 in vitro study by Honl., et al. investigated the use of plain and abrasive $\mathrm{WJ}$ as a cutting tool for endoprosthesis revision surgery. Five different (pure and abrasive) water pressure levels of 30, 40, 50, 60 and $70 \mathrm{MPa}$ were applied at two different angles $\left(30^{\circ}\right.$ and $\left.90^{\circ}\right)$ to cut samples of mid-diaphysis of human femora and bone cement. As shown in Figure 2.1, only bone cement was cut with pure WJ (PWJ). When using abrasive WJ (AWJ), significant higher cut depth were recorded in both bone and bone cement.
The study concluded that AWJ would be an alternative tool for cement removal. Additionally, AWJ is advantageous for revision of non-cemented prosthesis due to its possibility for localized cutting at interfaces.

A study in 2006 introduced Versajet WJ as an alternative to standard surgical excisional techniques for burn wounds. In the study, the Versajet WJ was able to sufficiently debride superficial partial thickness and mid-dermal partial thickness wounds for the subsequent placement of Biobrane. Additionally, the study demonstrated that the Versajet WJ is beneficial in the surgical treatment of superficial to mid-partial thickness burns in the face, hand and foot.

Another study conducted in 2007 reviewed the versatility of the Versajet WJ surgical tool in treating the deep and indeterminate depth face and neck burns. With ex-vivo histologic analysis of depth of debridement on human skin, the study confirmed that predictable and controlled depth of debridement could be obtained by adjusting the apparatus settings.

In 2010, Keiner., et al. published a paper with their research that took place from 1997 to 2009 . In the 12-year span, the authors performed 208 procedures on patients with various intracranial neurosurgical pathologies using WJ dissector with pressures ranging from 0.4 to 1.5 MPa. Müritz 1000, Helix Hydro-Jet, and ErbeJet 2 were used in these procedures. The surgeons evaluated the WJ devices used and recorded the differences and limitations in the various pathologies. The surgeons noted that the $\mathrm{WJ}$ apparatus was considered to be very helpful in 166 procedures $(79.8 \%)$ and helpful to some extent in 33 procedures (15.9\%). In eight (3.8\%) procedures, it was not helpful, and in one procedure $(0.5 \%)$, the usefulness was not documented by the surgeon. The authors concluded that WJ technology is safe and easy to apply in precise tissue dissection. They also noted in addition to such precision, the preservation of blood vessels and no greater risk of complications are possible with $\mathrm{WJ}$.

Not many studies have focused on using WJ technology on skin incisions. The research focused not only on the application of WJ technology on skin incision theoretically but also experimentally.

\section{Objectives}

The main objective of this research is to determine the precise depth of incision using WJ technology, verify the existing research 
results published in the literature. An analytical relationship is defined between the skin properties and the operating parameters of the $\mathrm{WJ}$ in various surgical procedures. These relationships include the skin thickness, its modulus of elasticity, the WJ pressure, the diameter of nozzle orifice, the nozzle standoff distance and the traverse speed of the WJ.

\section{Methodology}

The WJ machining has a variety of variables which independently determine the operating parameters of the skin incisions. These variables can be divided, based on system components, into four categories: Process, skin, nozzle and pump/intensifier characteristics.

\section{Process characteristics}

1. Depth of incision

2. Width of incision

3. Traverse speed

4. Feed rate

5. Stand-off distance

6. Pure or abrasive water

7. Continuous or discontinuous jet

\section{Skin characteristics}

1. Type of skin

2. Thickness

3. Hardness

4. Consistency

5. Body location

6. Age

7. Gender

8. Demographic

9. Specific Energy/Elastic Modulus

\section{Nozzle}

1. Nozzle structure and diameter

2. Orifice type and diameter

3. Pump and Intensifier

4. Direct drive

5. Hydraulic drive

6. Oil pressure

7. Pressure amplification

8. Pressure

9. Pump efficiency

10. Power
The mechanic of the waterjet skin incision is illustrated in Figure 1, and can be described as follows;

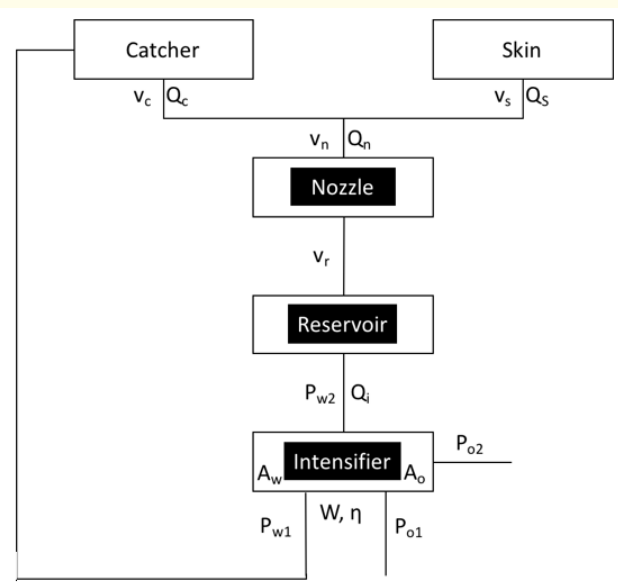

Figure 1: The mechanic of Waterjet incisions.

The skin characteristics determine the nozzle's requirement to make a certain incision. The two main components for a surgical incision are the width and the depth of incision. Before performing an incision, the surgical team must have these two factors determined. While the specific energy of many materials is known, it may not be known for organic materials such as the human skin. Based on the experimental results provided in previous study, the specific energy/ elastic modulus of the skin is determined. After determining the main components for making a skin incision, the relationship between the force of the water coming out of the nozzle $\left(\mathrm{F}_{\mathrm{w}}\right)$ and the force required for skin incision $\left(\mathrm{F}_{\mathrm{s}}\right)$ can be formulated as follows:

$\mathrm{F}_{\mathrm{w}}=\mathrm{F}_{\mathrm{s}}$

$\mathrm{F}_{\mathrm{w}} \mathrm{A}_{\mathrm{n}}=\mathrm{E} \cdot \mathrm{A}_{\mathrm{s}}$

where $\mathrm{E}$ is the specific energy/elastic modulus of the skin,

$A_{n}=\left\{\pi \cdot \frac{d_{n}^{2}}{4}\right\}$ and $A_{s}=\left\{D_{s} \cdot w_{s}\right\}$

are the areas of the nozzle and skin incisions

$\mathrm{d}_{\mathrm{n}}$ is the orifice diameter of the nozzle, Ds is the depth of incision and ws is the width of incision.

The relationship between the nozzle's orifice diameter and the width of incisions:

$\mathrm{w}_{\mathrm{s}}=\mathrm{d}_{\mathrm{n}} \cdot \mathrm{e}^{\mathrm{a} \cdot \mathrm{x}}$ 
where $\mathrm{a}$ is the taper index and $\mathrm{x}$ is the standoff distance of the nozzle.

The total Pressure energy required for the skin incisions:

$$
P E=E \cdot Q_{s}
$$

where $Q_{s}$ is the volume flow rate at which the WJ removes the skin, which is calculated as:

For skin cutting and debridement: $Q_{s_{c u t}}=D_{s} \cdot w_{s} \cdot s$

For skin drilling: $Q_{s_{-} d r i l l}=\frac{\pi \cdot d_{i}^{2} \cdot f}{4}$

where $s$ is the traverse speed, di is the insert diameter and $f$ is the feed rate.

The kinetic energy of the catcher is the remaining energy that is not absorbed by the skin incision:

$$
\mathrm{KE}_{\mathrm{c}}=\frac{1}{2} \cdot \mathrm{Q}_{\mathrm{c}} \cdot \mathrm{v}_{\mathrm{c}}^{2} \cdot \rho_{\mathrm{w}}
$$

where $\rho_{w}$ is the density of water, $Q_{c}$ is the volume flow rate of the residue water

The velocity at which the excess water is going to the catcher $\left(v_{c}\right)$ is:

$\mathrm{v}_{\mathrm{c}}=\sqrt{2 \cdot \mathrm{g} \cdot \mathrm{x}}$

where $\mathrm{g}$ is the gravity.

The kinetic energy of the WJ stream coming out of the nozzle is the sum of the pressure energy required to make the skin incision and the kinetic energy of the catcher:

$\mathrm{KE}_{\mathrm{n}}=\mathrm{PE}+\mathrm{KE}_{\mathrm{c}}$

It's also equal to:

Pure WJ: $\mathrm{KE}_{\mathrm{n}}=\frac{1}{2} \cdot \mathrm{Q}_{\mathrm{n}} \cdot \mathrm{v}_{\mathrm{n}}^{2} \cdot \rho_{\mathrm{w}} \cdot \mathrm{k}_{\mathrm{e}}$

Abrasive WJ: $\mathrm{KE}_{\mathrm{n}}=\frac{1}{2} \cdot \dot{\mathrm{m}}_{\mathrm{abr}} \cdot \mathrm{v}_{\mathrm{n}}^{2} \cdot \mathrm{k}_{\mathrm{e}}$

where $v_{n}$ is WJ velocity out of the nozzle, ke is loss coefficient and $m_{\text {abr }}$ is abrasive mass flow rate.

The relationship between the WJ velocity out of nozzle $\left(v_{n}\right)$ and velocity of the $\mathrm{WJ}$ stream at the $\operatorname{skin}\left(\mathrm{v}_{\mathrm{s}}\right)$ :

$\mathrm{v}_{\mathrm{s}}=\mathrm{v}_{\mathrm{n}} \cdot \mathrm{e}^{-\mathrm{a} \cdot \mathrm{x}}$

where $\mathrm{a}$ is the taper index and $\mathrm{x}$ is the standoff distance of the nozzle as in Equation (3)
The area and the shape of the orifice affect the flow of the water from the nozzle to the atmosphere. The different orifice types and the typical values of discharge $\left(\mathrm{C}_{\mathrm{d}}\right)$ and loss $\left(\mathrm{k}_{\mathrm{e}}\right)$ coefficients for water orifices are listed in Abdou [14].

The relationship between $Q_{n}$ and $v_{n}$

$\mathrm{Q}_{\mathrm{n}}=\mathrm{C}_{\mathrm{d}} \cdot \mathrm{A}_{\mathrm{n}} \cdot \mathrm{v}_{\mathrm{n}}$

The relationship between $\mathrm{v}_{\mathrm{n}}$ and the water pressure $\left(\mathrm{P}_{\mathrm{w}}\right)$ coming out of the nozzle :

$v_{n}=\sqrt{\frac{2 \cdot P_{w 2}}{\rho_{w}}}$

Re-arranging Equation 4.15, the pressure of the WJ is:

$$
P_{w 2}=\frac{v_{n}^{2} \cdot \rho_{w}}{2}
$$

The relationship between the velocity of the WJ flow coming out of the pump reservoir and the one coming out of the nozzle is calculated as follows:

$\mathrm{v}_{\mathrm{r}}=\mathrm{v}_{\mathrm{n}} \cdot \mathrm{e}^{-2 \cdot \beta \cdot \mathrm{L}_{\mathrm{n}}}$

where $L_{n}$ is the length of the nozzle, $\beta$ is the exponential constant, which is based on an exponential taper WJ nozzle design [14] and $d_{o}$ is the larger diameter of the nozzle:

$\beta=\frac{-\ln \left(d_{n} / d_{o}\right)}{L_{n}}$

The pressure ratio $\left(\mathrm{r}_{\mathrm{p}}\right)$ between the water outlet pressure $\left(\mathrm{P}_{\mathrm{w} 2}\right)$ and the oil inlet pressure $\left(\mathrm{P}_{01}\right)$ is equal to the inverse proportion of the oil inlet area $\left(A_{0}\right)$ and the water inlet area $\left(A_{w}\right)$ :

$r_{p}=\frac{P_{w 2}}{P_{o 1}}=\frac{A_{o}}{A_{w}}$

The WJ flow rate out of the intensifier $\left(Q_{\mathrm{i}}\right)$ is equal to the $\mathrm{WJ}$ flow rate coming out of the nozzle $\left(Q_{n}\right)$. Thus, the Power $(W)$ is determined by the water pressure coming out of the intensifier $\left(\mathrm{P}_{\mathrm{w} 2}\right)$, the efficiency of the intensifier $\left(\eta_{i}\right)$ and the flow rate $\left(Q_{i}\right)$ as follows:

$W=\frac{P_{w 2} \cdot Q_{i}}{\eta_{i}}$

These relationships are robust enough to be applied to some existing WJ apparatus: top three WJ brands in surgical procedures: ErbeJet 2, Helix HydroJet and VersaJet. 


\section{Derived analytical model}

From Equations (1)-(15), the pressure for pure and abrasive WJ:

$$
\begin{aligned}
& \boldsymbol{P}_{p w j}=\frac{\rho_{w}}{2} \cdot\left(\frac{8 \cdot Q_{s}}{c_{d} \cdot \pi \cdot d_{n}^{2} \cdot \rho_{w} \cdot k_{e}}\right)^{\frac{2}{3}} \\
& \boldsymbol{P}_{a w j}=\frac{4 \cdot D_{s} \cdot(1+R)^{2} \cdot E \cdot d_{i} \cdot s}{c_{d} \cdot \pi \cdot d_{n}^{2} \cdot R \cdot k_{e}}
\end{aligned}
$$

Re-arranging Equations (16) and (17), the depth of incision for pure and abrasive WJ:

$$
\begin{gathered}
D_{s_{-} p w j}=\frac{c_{d} \cdot \pi \cdot v_{n} \cdot \rho_{w} \cdot d_{n}^{2} \cdot k_{e}}{8 \cdot E \cdot w_{S} \cdot s} \\
D_{s_{-} a w j}=\frac{c_{d} \cdot \pi \cdot v_{n}^{3} \cdot \rho_{w} \cdot d_{n}^{2} \cdot R \cdot k_{e}}{8 \cdot(1+R)^{2} \cdot E \cdot d_{i} \cdot s}
\end{gathered}
$$

The velocity of the WJ coming out of the nozzle for pure and abrasive WJ:

$$
\begin{gathered}
v_{n_{-} p w j}=\sqrt[3]{\frac{8 \cdot Q_{s} \cdot E}{c_{d} \cdot \pi \cdot d_{n}^{2} \cdot \rho_{w} \cdot k_{e}}} \\
v_{n_{-} a w j}=\sqrt{\frac{8 \cdot D_{s} \cdot(1+R)^{2} \cdot E \cdot d_{i} \cdot s}{c_{d} \cdot \pi \cdot d_{n}^{2} \cdot \rho_{w} \cdot R \cdot k_{e}}}
\end{gathered}
$$

where $\mathrm{R}$ is ratio of mass flow rate of the abrasive material to mass flow rate of the water.

Making a skin incision using abrasive WJ is rare due to the minimal pressure necessary to incise the skin. However, the abrasive $\mathrm{WJ}$ equations could possibly be used in making incisions in other organic materials such as bones and bone cement.

\section{Model verification}

The developed analytical model is tested using the experimental work done by Honl., et al in 2004 where WJ technology is used as a cutting tool for end prosthesis revision surgery. The specific energy of the bone cement is calculated from the given parameters. At $30 \mathrm{MPa}$ WJ pressure, the specific energy is calculated to be 1.43 $\mathrm{x} 105 \mathrm{MPa}$. This value is then used to verify the developed analytical model; The calculated depth of cut compared to the published measurements, are shown in the Table (shown below). The percent error between the measured and calculated values ranges between $2 \%$ (for 50 and $60 \mathrm{MPa}$ WJ pressure) and 11\% (for $40 \mathrm{MPa}$ WJ pressure).

\begin{tabular}{|l|c|c|c|}
\hline Pressure & $\begin{array}{c}\text { Measured Depth } \\
\text { of Cut }\end{array}$ & $\begin{array}{c}\text { Calculated } \\
\text { Depth of Cut }\end{array}$ & $\begin{array}{c}\text { Percent } \\
\text { Error }\end{array}$ \\
\hline $30 \mathrm{MPa}$ & $\sim 0.75 \mathrm{~mm}$ & - & - \\
\hline $40 \mathrm{MPa}$ & $\sim 1.10 \mathrm{~mm}$ & $1.23 \mathrm{~mm}$ & $11 \%$ \\
\hline $50 \mathrm{MPa}$ & $\sim 1.75 \mathrm{~mm}$ & $1.72 \mathrm{~mm}$ & $2 \%$ \\
\hline $60 \mathrm{MPa}$ & $\sim 2.21 \mathrm{~mm}$ & $2.26 \mathrm{~mm}$ & $2 \%$ \\
\hline $70 \mathrm{MPa}$ & $\sim 3.00 \mathrm{~mm}$ & $2.85 \mathrm{~mm}$ & $5 \%$ \\
\hline
\end{tabular}

Table: Measured vs. Calculated Depth of Cut for Bone Cement.

\section{Conclusion}

This research is able to explore the theoretical and analytical aspects of WJ technology that help us understand it better without the costs or the risks. By providing the analytical relationship between the skin properties, or any material to be incised, and the operating parameters of the WJ apparatus, one can simulate any procedure in advance to see the results before physically operating on the subject. Further research may include verifying the developed model using porcine skin, since its material properties are similar to that of human skin. Additionally, verifying the model using a WJ apparatus that is specified for medical procedures with lower water pressure is recommended for more accurate results.

\section{Bibliography}

1. LNH Daniel Smeak. "Core Surgical Skills: Basic Instrument Use". (2011).

2. AS a H Feussner. "Biomedical Engineering in Gastrointestinal Surgery”. Elsevier, (2017).

3. RD Petra Wilder-Smith., et al. "Incision Properties and Thermal Effects of Three CO2 Lasers in Soft Tissue". Oral Surgery, Oral Medicine, Oral Pathology, Oral Radiology, and Endodontology 79.6 (1995): 685-691.

4. NAK Khawar Saeed Jamali., et al. "Diathermy Incisions vs. Surgical Scalpel Incisions; Outcome in General Surgery". The Professional Medical Journal 22.11 (2015): 1520-1524.

5. ID Dilip Kumar Dutt. "The Harmonic Scalpel”. The Journal of Obstetrics and Gynecology of India 66.3 (2016): 209-210.

6. MMJ Edward., et al. "A Single-blind Controlled Study of Electrocautery and Ultrasonic Scalpel Smoke Plumes in Laparoscopic Surgery". Surgical Endoscopy 26.2 (2012): 337-342. 
7. SH Pavol Hreha., et al. "Water Jet Technology Used in Medicine". Technical Gazette 17.2 (2010): 237-240.

8. J Beard. Technology: Water Jet Puts Surgeons at the Cutting Edge. NewScientist (1994).

9. G Yildirim. "Using Water Jet Technology to Perform Skin Surgery". (2003).

10. HE S Hans-Oliver., et al. "Debridement of burn wounds with a water jet surgical tool”. Burns 32 (2006): 64-69.

11. RR Matthias Honl., et al. "The Water Jet as a New Tool for Endoprosthesis Revision Surgery - An in vitro Study on $\mathrm{Hu}$ man Bone and Bone Cement". Bio-Medical Materials and Engineering 13 (2003): 317-325.

12. DBM Tenenhaus and HO Rennekampff. "Treatment of deep partial thickness and indeterminate depth facial burn wounds with water-jet debridement and a biosynthetic dressing". Injury, International Journal of The Care of the Injured (2007): 7.

13. D Keiner., et al. "Water jet dissection in neurosurgery: An update after 208 procedures with special reference to surgical technique and complications". Neurosurgery 67.2 (2010): 342- 354.

14. G Abdou. "Analysis of Velocity Control of Waterjets for Waterjet Machining". presented at the Waterjet Cutting West, Lost Angeles, California (1989).

15. M Hanna. "Waterjet Technology in Skin Incision". N Atalla, Ed., ed, (2018).

Volume 3 Issue 8 August 2019

C All rights are reserved by Abdou George. 